\title{
Impact of Clinical Pharmacist Interventions on Improving Nurses' Practice of Tube Occlusion Management in Patients on Enteral Feeding Tubes (EFT)
}

\author{
Athira BM
}

PhD Scholar, Shri Jagdishprasad Jhabarmal Tibrewala University Vidyanagari, Jhunjhunu, Rajasthan, India

\section{Abstract}

\begin{abstract}
A high number of patients in the hospital setting are dependent on Enteral Feeding Tubes (EFT) due to varying reasons like disease related disabilities or swallowing difficulties. Tube occlusion is a very common complication occurring in patient on EFT. The nurses are many times unaware for the proper techniques to be followed in case of tube occlusion events. This study aims at assessing the routine practices followed by nurses related to management of tube occlusions and the impact of training provided by clinical pharmacist on the same. A prospective interventional study was carried out in a tertiary care hospital of Kerala state of India. Clinical pharmacist led training programmes and guideline development was performed during the intervention phase. The pre interventional and post interventional data were compared and statistically analyzed using SPSS version 13. In the post intervention phase proper use of warm water and use of alkalinized solution of pancreatic enzymes increased significantly $(\mathrm{P}<0.001)$. Changing of the tube reduced from $74.6 \%$ to $21.4 \%$ which was significant $(\mathrm{p}<0.001)$. The study shows that clinical pharmacist interventions through training, auditing and implementation of guidelines can significantly improve the tube occlusion management in patients on EFT and ensure quality and safety of therapy.
\end{abstract}

Keywords: Tube occlusion, Eneteral Feeding Tube (EFT), Clinical pharmacist, Nurses.

Copyright (C) 2020 The Author(s): This is an open-access article distributed under the terms of the Creative Commons Attribution 4.0 International License (CC BY-NC 4.0) which permits unrestricted use, distribution, and reproduction in any medium for non-commercial use provided the original author and source are credited.

\section{INTRODUCTION}

A high number of patients in the hospital setting are dependent on Enteral Feeding Tubes (EFT) due to varying reasons like disease related disabilities or swallowing difficulties. The administration of feed and drugs through enteral feeding tube is mostly the responsibility of nurses. Nurses are required to have adequate knowledge and skills to perform these duties without any error. Maintaining the patency of enteral tubes is a challenge faced by nurses. Tube occlusion is a very common complication occurring in patient on EFT. The tubes get occluded due to various reasons like narrow orifice of tube, cyclical administration of feed, remaining of stagnant feed in tube, reaction of tube material with feed, improper administration of drugs and formation of feed precipitate due to contact with acidic fluid. Care should be taken to avoid such circumstances. In case if occlusion occurs even after careful management, proper techniques should be applied in de occluding the tubes. The most efficient method is flushing with warm water. Carbonated drinks were once heavily favored as enteral feeding tube flushes, but trials have demonstrated that warm water performs as well as other fluids tested as an enteral feeding tube flush. It should be noted that acidic flushes such as cola can exacerbate tube occlusion by causing feed to coagulate or protein to denaturize. Water is the most appropriate fluid with which to flush and is as effective as any flush for reducing the formation of, and for clearing previously established, tube occlusions. A pulsatile flushing action should be used to create turbulence within the inner lumen of the enteral feeding tube, cleaning the inner walls more effectively [1]. In case of occlusions not cleared by warm water, use of pancreatic enzymes is a good option. Pancreatic enzyme containing capsules like Creon can be opened and the contents can be mixed with a solution of sodium bi carbonate tablet in water. Such alkalinized solution of pancreatic enzyme is proven to be effective in unblocking [2]. Changing of tubes should be the last option management occlusions because unnecessary tube changes will increase the morbidity and cost of treatment. 
The nurses are many times unaware for the proper techniques to be followed in case of tube occlusion events. This will affect the patients' health outcomes and will also cause financial burden. Clinical pharmacist as a responsible professional has to make sure that the practices are correctly followed in a hospital. This study aims at assessing the routine practices followed by nurses related to management of tube occlusions and the impact of training provided by clinical pharmacist on the same.

\section{METHODOLOGY}

\section{Study site and design}

A prospective interventional study was carried out in a tertiary care hospital of Kerala state of India.

\section{Study period and method}

For the initial assessment data was collected for a period of 3 months. All the patients put on any type of enteral feeding tube during the study period were included. The clinical pharmacist prospectively followed all the selected patients on a daily basis and the occlusion events were identified. The nurses were also asked to report such events. When a tube occlusion is reported the clinical pharmacist will analyze strategies followed in the management of the occlusion, by direct observation and also by interviewing the nurses. The main focus was on detecting the following:

- What was the first step taken when a tube occlusion occurred?

- Whether the tube was changed as soon as the occlusion occurred

- Whether de blocking was tried

- Which method was used in the de blocking of tube

These were noted down in a specially designed data collection form for each patient and the data was compiles at the end of the initial assessment period.

\section{Intervention and post intervention study}

Clinical pharmacist led training was conducted during the intervention phase to educate the nurses on the proper methods to be followed to manage the tube occlusions. The classes were taken using slide presentation and also by demonstration of various techniques. It was made sure that all nurses attended the classes. A guideline was also prepared and implemented with the permission of the management. The guideline was distributed to all the wards and ICUs.

A post interventional study was carried out for 4 months where the pre interventional study procedure was repeated. After this period the data collected was compared with the data obtained before intervention.

\section{DATA ANALYSIS}

Each parameter was compared between the two sets of data to identify the impact of interventions. The statistical analysis was carried out using SPSS (Statistical Package for Social Sciences) version 13.

\section{RESULTS}

160 patients were included in the pre intervention study and 245 patients in the post intervention study. The total number of tube occlusions was 67 before intervention and 73 after intervention. $26 \%$ of nurses used to directly report to the physician when the tube gets blocked without trying to manage by them. This decreased to $5 \%$ after interventions. Most of the nurses started to manage the events on their own. The change was statistically significant $(\mathrm{P}<0.05)$. Proper use of warm water for de occluding tubes was followed by only $23.9 \%$ which increased to $87.9 \%$ in the post intervention phase $\mathrm{p}<0.001)$. The use of carbonated beverages had not been followed in the institution and no such practice was identified before or after intervention phase. There was no awareness about the use of alkalinized solution of pancreatic enzymes for de blocking tubes. Therefore this method was not utilized in any case before intervention. However after intervention the use of this method was found in $33.33 \%$ of cases $(p<0.001) .12 \%$ of nurses reported to directly change the tube without applying de blocking techniques which decreased to $1 \%$ after interventions $(p<0.05)$. The total number of tube changes was found to be 50 out of 67 in the pre intervention group. That is the tube was changed in $74.6 \%$ of cases of tube occlusion. In the post intervention study tube changes was only in $21.2 \%$ of all the cases of occlusion which was a significant decrease $(\mathrm{p}<0.001)$.

\section{DISCUSSION}

The study results reveal that the knowledge related to management of EFTs and de occlusion techniques is very limited among the nurses. The lack of knowledge leads to improper practices that will affect the quality of care and will result is adverse health and financial outcomes in patients. Clinical pharmacists can intervene through educational and review programmes to minimize the errors and improve the outcomes. When the nurses were trained and guidelines were implemented the practices improved significantly.

The incidents of tube occlusion and factors related to it have been discussed in many previous studies. 67 tube obstruction events were observed from 160 patients in pre intervention phase and 73 obstruction events were observed from 245 patients in post intervention phase in the present study. This is similar to the study by Fernanda et al. who identified 136 cases of obstructions in 374 patients. They established positive relationship of tube obstructions with mixing drugs together, incorrect reconstitution of tablets, improper flushing, concurrent administration of drugs and feed and unplanned withdrawal of tube [3]. Gorzoni et al. found that if proper time interval between feed and drugs is not kept there will be increased risk of precipitation and tube obstruction. Proper flushing of tube is also important to prevent blockage [4]. Isabela et al. found that patients given more than 5 drugs through 
EFT and those having more than 13 administrations per day through EFT have greater risk of getting their tubes changed [5]. In current study also such factors may have contributed to the high number of occlusion events.

Directly changing the tube without applying unblocking techniques was a common practice before intervention. The total tube changes significantly reduced from $74.6 \%$ to $21.2 \%$ after intervention with $\mathrm{p}<0.001$. The proper use of warm water for unblocking could be significantly increased. The nurses and doctors were not aware about the other effective methods for unblocking the enteral tubes. Nurcan Uysal et al. in their study assessed the nurses and found that $38 \%$ of nurses used to report to physician when occlusion occurred, $25.5 \%$ used to directly remove and replace the tube and only $9.2 \%$ said they would try to unblock by flushing [6]. This shows that the lack of correct practice of unblocking tube is lacking worldwide. The use of pancreatic enzymes for more complicated occlusions was introduced during the intervention phase and $33.33 \%$ cases were applied with this method. The study done by Stumpf et al. provides proof on the efficacy of alkalinized solution of pancreatic enzyme preparations in de blocking the occluded tubes [7].

Clinical pharmacist led programmes can be introduced as effective approach in optimizing the management of therapy through EFTs. The interventional study carried out by Bemt et al. identified decreased number of tube occlusions as an outcome of pharmacist interventions. They implemented various interventional approaches like pharmacist led review system, 'do not crush' labels, setting up of data base etc in two teaching hospitals of Netherlands and reduction in number of tube occlusions was observed in one of the hospitals [8].

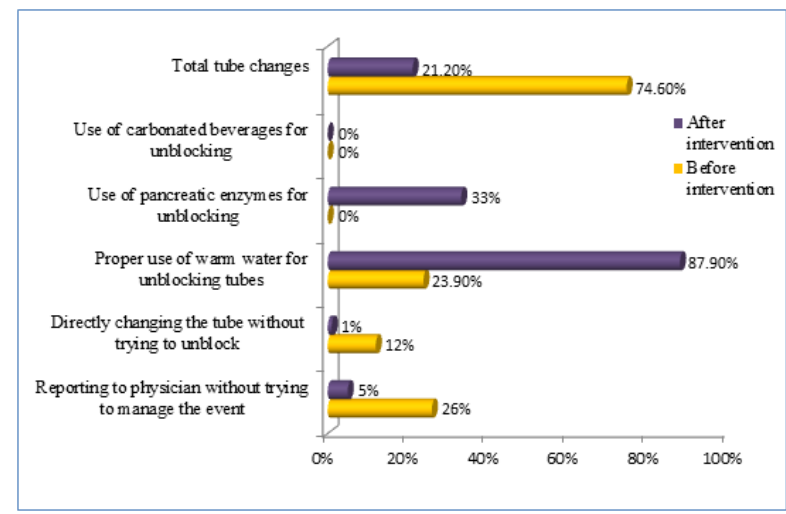

Fig-1: Impact of clinical pharmacist interventions on various factors related to tube occlusion management by nurses

\section{CONCLUSION}

Managing patients on EFTs is a process requiring adequate knowledge and skills. Nurses as professionals responsible for administration of feed and drugs should be well aware of the standard protocols and procedures related to this. Unfortunately most of the nurses are having very poor knowledge related to management of EFTs and this leads to various complications. One of the common incidents in patients on EFT is tube occlusion. Majority of nurses do not handle occlusion events appropriately. Clinical pharmacist interventions through training, auditing and implementation of guidelines can significantly improve this process and ensure quality and safety of therapy.

\section{REFERENCES}

1. White, R., \& Bradnam, V. (2015). Handbook of drug administration via enteral feeding tubes. Pharmaceutical press.

2. Stumpf, J. L., Kurian, R. M., Vuong, J., Dang, K., \& Kraft, M. D. (2014). Efficacy of a Creon delayed-release pancreatic enzyme protocol for clearing occluded enteral feeding tubes. Annals of Pharmacotherapy, 48(4), 483-487.

3. Gimenes, F. R. E., Pareira, R. A., Horak, A. C. P., Oliveira, C. C., Reis, A. M. M., da Silva, P. I. C. D. S., ... \& Atila, E. (2017). Medication incidents related to feeding tube: a cross-sectional study. African Journal of Pharmacy and Pharmacology, 11(27), 305-313.

4. Gorzoni, M. L., Della Torre, A., \& Pires, S. L. (2010). Drugs and feeding tubes. Revista da Associação Médica Brasileira, 56(1), 17-21.

5. Heineck, I., Bueno, D., \& Heydrich, J. (2009). Study on the use of drugs in patients with enteral feeding tubes. Pharmacy world \& science, 31(2), 145-148.

6. Uysal, N., Sari, H. Y., Gok, D. D., \& Maslak, O. O. (2016). Evaluation Nurses' Practices for medication administration via enteral feeding tube. International Journal of Caring Sciences, 9(3), 1058-1065.

7. Stumpf, J. L., Kurian, R. M., Vuong, J., Dang, K., \& Kraft, M. D. (2014). Efficacy of a Creon delayed-release pancreatic enzyme protocol for clearing occluded enteral feeding tubes. Annals of Pharmacotherapy, 48(4), 483-487.

8. Van den Bemt, P. M. L. A., Cusell, M. B. I., Overbeeke, P. W., Trommelen, M., Van Dooren, D., Ophorst, W. R., \& Egberts, A. C. G. (2006). Quality improvement of oral medication administration in patients with enteral feeding tubes. BMJ Quality \& Safety, 15(1), 44-47. 